\title{
Research on the manufacturing process oriented customer satisfaction evaluation
}

\author{
Qing Liu ${ }^{1}$, Gang Huang ${ }^{2}$, Shanshan $\mathrm{Yu}^{3}$ \\ 1.2.3 College of Quality and Safety Engineering \\ lqcjlu@163.com,835136654@qq.com,1660296743@qq.com
}

\begin{abstract}
Keywords: Complete Manufacturing Process; Customer satisfaction; AHP; ANFIS
Abstract. This paper presents a method to measure the manufacturing process oriented customer satisfaction. The method combines AHP and fuzzy neutral network, firstly, the paper defines the concept of Complete Manufacturing Process as foundation of the method, and constructs customer satisfaction indicator system, then weights of the indicators are used to choose inputs and improve the membership function of Fuzzy Neural Network. The improved neural network is trained by customers habit and preference so as to evaluating customer satisfaction. At last, numerical example is presented to illustrate the reasonability and feasibility of the method.
\end{abstract}

\section{Introduction}

The purpose of service is to make customers as satisfied as possible. If the production process of manufacturing companies can be considered as a service provided to the customer [1]. To better serve the customer, it is necessary to conduct customer satisfaction evaluation for the production process so as to fully consider how customer involvement and experience would the impact on the manufacturing process. Customer satisfaction comes from the degree to which these factors are met [2]. At the same time, behavioral traits of customers are truly the important Basis for evaluating and improving satisfaction [3]. Therefore, production process oriented customer satisfaction measuring should relate production process with customers, and take production process factors, customer behavior and customer satisfaction into consideration as a whole. However, traditional customer satisfaction only aims at part of aspects of the manufacturing process.

In this paper, we propose a production process oriented customer satisfaction evaluation method. With this method, we establish the mapping relationship between the factors of production process and the customer satisfaction level, quantify the satisfaction value according to the customer behavior habit, and further more adjust and improve the unqualified customer satisfaction according to enterprise strategy and expectation.

\section{Problem description and model establishment}

\section{A Determination of the key factors in the production process and evaluation}

The expanded complete production process centers on the manufacturing process, extending to the pre-production supplier selection process and the post-production logistics and distribution process. On the basis of determining the scope of the production process, we must first refine and define the key factors, and establish the evaluation index system.

This article divides the indicators of the expanded production process into two categories: direct production process indicators and indirect production process indicators. Direct production process indicators are the traditional production scheduling results, and indirect production process indicators cover materials, logistics, production control and so on. The detailed definition is as follows:Direct Manufacturing Process Indicators - Scheduling results index (SRI), which include makespan SR1; tardiness SR2; earliness SR3; fulfill rate (current inventory level ) SR4; delivery lot and batch SR5. Indirect Production Process Indicators ---- Production \& Supply Chain Index (PSI): Raw Material Supplier PS1; Part Outsourcing PS2; Logistics Method PS3; Environmental and Safety Certification PS4; Workshop Production Control Method PS5. 
The evaluation index of these indexes belongs to the multi-attribute evaluation problem. The commonly used 9-level scale AHP [4] method is adopted in this paper. With this method, that the weights of the index in the index system ${ }^{w_{i}}$ are got by establishing comparison matrix, then the weight is used as the degree of effect on customer satisfaction in this paper.

\section{B Customer Satisfaction Rating / Production Factors and Customer Satisfaction Mapping}

Production process indicators have a variety of satisfaction possibilities. Simple "satisfaction" or "not satisfaction" can not cover all the evaluation status. This paper divides the satisfaction evaluation of indicators into five levels: very poor, poor, medium, good and excellent. And quantitative methods are used to reflect the differences in the satisfaction of the indicators of the production process. Respectively, corresponding to the value of 1-5,1-very poor, 2-poor, 3-medium, 4-good, 5 excellent. What's more, there are several options for each indicator. For example, raw material suppliers have three categories of "partners", "long-term suppliers" and "new suppliers." However, customers of different grades / types have different options for the same indicators Satisfaction ratings. For small customers, the new suppliers can achieve "good" or "excellent" evaluation, but for the long-term cooperation of large customers, the new suppliers may get "bad" rating because of the quality, availability and other aspects of the uncertainty. If customers have an overall high value of the various indicators, the higher the level of satisfaction would also be high.

The mapping process from the results of a complete production process to the quantitative values of customer satisfaction needs to take into account the weight of the indicator, the type of customer, and the customer's decision-making habits and mindset. This paper uses fuzzy neural network to describe this process.

Fuzzy neural network is a kind of fuzzy reasoning system based on neural network. Fuzzy neural network is suitable for expressing fuzzy or qualitative knowledge, its reasoning way is similar to human thinking mode, it can handle the problems of uncertainty and non-linearity, and has the characteristics of adaptive learning. The ANFIS (Adaptive Neural Network based Fuzzy Interferences System) is based on the improved fuzzy neural network proposed by Wang Shitong [5], which is combined with the aforementioned AHP index weights. There are four layers in total, which are input layer, membership function generation layer, inference layer, and anti-blur layer. Training data is obtained based on survey data and manufacturing companies have completed orders and customer feedback from the situation.

\section{Customer satisfaction adjustments based on the factors of production and weight application examples}

When delivery and quality are no longer the only criteria for customer satisfaction, and take into account all aspects of a complete production process instead. The evaluation of customer satisfaction based on the above method can make clear the role played by each factor of production process in making customer satisfaction; estimate customer satisfaction response before delivery of production arrangement result; and when possible, adjust the cost of indicators, customer expectations, development strategies to change customer satisfaction, so as to make customer satisfaction as high as possible, or to give beyond desird satisfaction to the desired long-term customers. The steps of the proposed method are as follows: 


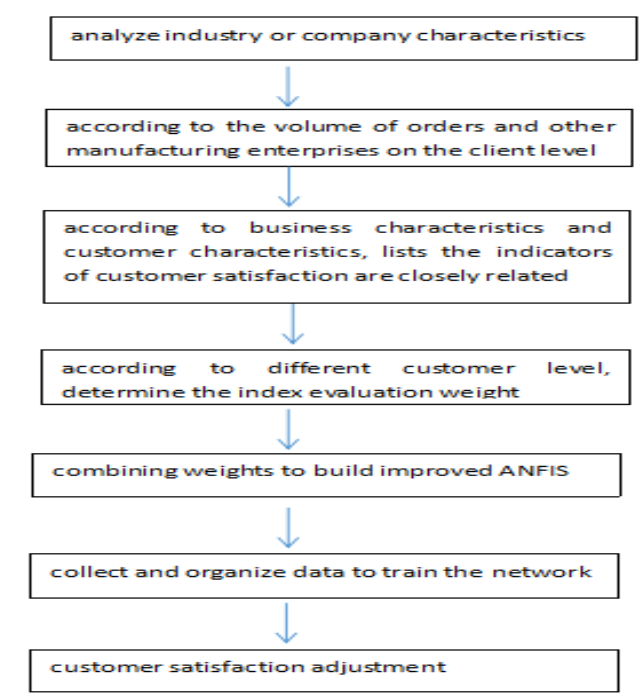

Figure. 1 Step diagram of the proposed method

\section{application examples}

\section{A satisfaction quantified}

In this paper, ANFIS is combined with indicator weight, which is combined with client category. Therefore, the weight and network obtained by instance verification correspond to a certain customer category to simulate customer behavior, Satisfaction responses to the results of manufacturing and shipping arrangements are quantified. And further adjust the customer satisfaction according to the corporate strategy, and ultimately improve customer satisfaction.

Suppose factory $\mathbf{M}$, there are long-term, medium and short-term three types of customers. this article sent questionnaires to mid-level customers to obtain customer feedback data for network training. In this example, the original indicators are the above-mentioned indirect indicators PS1-PS5 and the direct indicators SR1-SR4. According to the method steps, AHP method is firstly used for weight evaluation to obtain the original indicator weight values as follows:

Table. 1 Original Indicator Weigh

\begin{tabular}{lllllllllll}
\hline & PS1 & PS2 & PS3 & PS4 & PS5 & SR1 & SR2 & SR3 & SR4 & Weights \\
\hline PS1 & 1 & 2 & 4 & 4 & $1 / 3$ & $1 / 4$ & $1 / 4$ & 4 & 2 & 1.228 \\
PS2 & $1 / 2$ & 1 & 3 & 4 & $1 / 6$ & $1 / 7$ & $1 / 6$ & 2 & $1 / 6$ & 0.586 \\
PS3 & $1 / 4$ & 1 & 1 & 4 & $1 / 6$ & $1 / 7$ & $1 / 7$ & 2 & $1 / 5$ & 0.473 \\
PS4 & $1 / 5$ & $1 / 5$ & $1 / 6$ & 1 & $1 / 4$ & $1 / 5$ & $1 / 5$ & 1 & $1 / 5$ & 0.288 \\
PS5 & 2 & $1 / 3$ & 5 & 3 & 1 & $1 / 7$ & $1 / 5$ & 4 & $1 / 4$ & 0.867 \\
SR1 & 4 & 5 & 6 & 6 & 5 & 1 & 1 & 6 & 5 & 3.625 \\
SR2 & 3 & 4 & 5 & 5 & 4 & 1 & 1 & 5 & 2 & 2.840 \\
SR3 & $1 / 4$ & $1 / 2$ & $1 / 3$ & 1 & $1 / 4$ & $1 / 7$ & $1 / 4$ & 1 & $1 / 2$ & 0.384 \\
SR4 & $1 / 6$ & 4 & 5 & 5 & 4 & $1 / 7$ & 3 & 2 & 1 & 1.567 \\
\hline
\end{tabular}

In order to control the number of inputs, the AHP weights are used to screen the above indicators to be evaluated, and the indicators with too small weight values are excluded. From the observation, the weight of PS4 is only 0.288 , which is relatively small compared with other indicators. Indicator PS4 is "Environmental and Safety Certification", which can be regarded as having little impact on customer satisfaction currently. Therefore, it is not considered for further consideration as inputs of ANFIS. AHP weights of remaining indicators are normalized as follows: 
Table. 2 Normalized weights

\begin{tabular}{ccc|ccccc}
\hline PS1 & PS2 & PS3 & PS5 & SR & SR & SR & SR \\
& & & & 1 & 2 & 3 & 4 \\
\hline 0.1 & 0.0 & 0.0 & 0.0 & 0.3 & 0.2 & 0.0 & 0.1 \\
06 & 51 & 41 & 75 & 13 & 45 & 33 & 35 \\
\hline
\end{tabular}

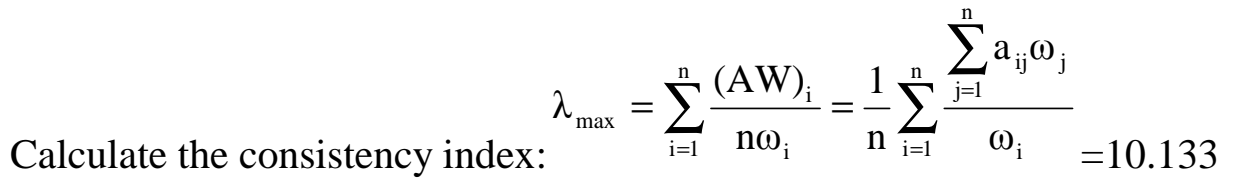

C.I. $=\frac{\lambda_{\max }-n}{n-1}=0.0 .142$, C.R. $=\frac{\text { C.I. }}{\text { R.I. }}=0.097<0.1$ is got, which is in line with the consistency of judgment

The options for each indicator are as follows. As mentioned earlier, we still set the rating of each indicator to five levels, represented by the numbers 1-5, although the number of options is less than5

Table. 3 Indicator Options

\begin{tabular}{|c|c|c|}
\hline Index & & Optional \\
\hline $\begin{array}{ll}\text { Raw } & \text { material } \\
\text { suppliers } & \end{array}$ & PS1 & Partner, long-term supplier, new supplier \\
\hline $\begin{array}{l}\text { Part outsourcing } \\
\text { situation }\end{array}$ & PS2 & $\begin{array}{l}\text { No outsourcing of components, providing outsourcers } \\
\text { and new outsourcers with consistent and stable service }\end{array}$ \\
\hline Shipping methods & PS3 & $\begin{array}{l}\text { 3PL, own logistics, a number of manufacturers } \\
\text { collaboration }\end{array}$ \\
\hline $\begin{array}{l}\text { Workshop } \\
\text { production control }\end{array}$ & PS5 & Effective organization, still valid, generally effective \\
\hline Drag the time & SR1 & No, short, medium, longer \\
\hline Advance time & SR2 & No, short, medium, longer \\
\hline Satisfaction rate & SR3 & $\begin{array}{l}\text { Less existing inventory, existing inventory to meet a } \\
\text { small number of orders, the existing stock to meet most } \\
\text { orders }\end{array}$ \\
\hline $\begin{array}{l}\text { Delivery volume } \\
\text { and batch }\end{array}$ & SR4 & $\begin{array}{l}\text { Small batch delivery according to customer needs, the } \\
\text { larger delivery volume, a settlement }\end{array}$ \\
\hline
\end{tabular}

For example, a mid-level customer order production arrangement results (new suppliers, providing continuous and stable service outsourcers, multiple manufacturers collaboration, effective organization, 0,0 , the existing stock to meet a small part of the order, one time clearing), the customer is "satisfied" with the result based on the historical data of the manufacturing plant or the questionnaire. In organizing the network training data, this "production arrangement result" and "satisfaction level" pair can be quantified as (4,3,2,5,5,5,5,5,5,5,3,80) .

ANFIS is established for the data example with 8 inputs, each representing one indicator, and each node has 5 sub-nodes in its own membership function layer. The reasoning layer contains 5 nodes according to the satisfaction level to be drawn, and the last layer is a node output layer.

We use a set of generated data to train this network. The purpose of training is to adjust membership function parameters and fuzzy rules so that control the error between the system output value and the given value within the allowable range. This paper chooses enough steps 3000 for training. In the process, the error first decreases gradually with the increase of training times, and then gradually increases after it reaches about 0.015. From the relationship between error and training steps to find the minimum error $(0.0115,2770)$, the corresponding membership function parameters is the network of the best parameters.

\section{B Customer Satisfaction Adjustment}

It should be pointed out that for large-scale production plants with a large number of customers, the same indicator would be of different importance to different types of customers, so the establishment 
of the weight system should be defined according to the enterprise customer type or customer category corresponding. And customer satisfaction also can be improved by adjusting the evaluation index. as the adjustment costs. Before adjusting, the adjustment cost of the indicator and the weight of the indicator may be comprehensively taken into consideration. The ANFIS can be used to make the pre-adjustment to forecast the situation of cost and satisfaction adjusting result. After the ideal result is obtained, the final production arrangement result is delivered to the customer, thereby improving customer satisfaction.

\section{Conclusion and Outlook}

Improving customer satisfaction is related to the long-term development of enterprises and is also the foundation of service-oriented manufacturing. Based on the full production process, this paper targets at measuring and adjusting customer satisfaction by combining the AHP and the fuzzy neural network. The integration of the evaluation process and the customer / customer decision-making characteristics is reflected in three aspects: firstly, the two-by-two judgment matrix is constructed by the scoring of customers; secondly, the combination of indicator weights of production process and ANFIS highlights the importance of different indicators in the process of quantification of satisfaction Sexuality; Finally, the network's training data comes from the customer's decision-making habits.

\section{Acknowledgement}

This research is supported by Natural Science Foundation of Zhejiang Province, No.LQ15G010005

\section{References}

[1] Li Hao, Ji Yangjian, Qi Guoning, Gu Xinjian, Tang Renzhong. The Connotation, Theory and Key Technology System of Manufacturing and Service Integration [J]. Computer Integrated Manufacturing Systems, 2010,16 (11), 15-23.

[2] Duan Xuan, Zhu Xichuan, catkins. Hierarchical Analysis of Customer Satisfaction in Machinery Manufacturing Enterprises [J]. Mechanical Design and Research, 2011,27 (6): 1-4

[3]Ling Lia, John B. Forda, Xin Zhaib and Li Xua. Relational benefits and manufacturer satisfaction: an empirical study of logistics service in supply chain[J]。 International Journal of Production Research, 2012,50(19): 5445-5459

[4] T L Satty. The Analytic Hierarchy Process--Planing, Priority Setting, Resource Allocation. New York:Mc Graw Hill,Lnc.1980:287

[5] Wang Shitong. Fuzzy system, fuzzy neural network and application design [M]. Shanghai: Shanghai Science and Technology Literature Publishing House, 1998 